\title{
MAPEAMENTO SISTEMÁTICO DE LITERATURA SOBRE ESTUDOS DE INTERFACES DE USUÁRIO EM TECNOLOGIA ASSISTIVA
}

\section{SYSTEMATIC REVIEW OF THE LITERATURE ON STUDIES OF USER INTERFACES IN ASSISTIVE TECHNOLOGY}

\author{
Vinicius Kruger da Costa ${ }^{1}$, Esp. \\ Andréia Sias Rodrigues ${ }^{2}$, M. Sc. \\ Rafael Cunha Cardoso ${ }^{3}$, M. Sc. \\ Tatiana Aires Tavares ${ }^{4}$, D. Sc.
}

(1) Centro de Desenvolvimento Tecnológico - Universidade Federal de Pelotas - CDTec-UFPel e-mail: viniciusdacosta@pelotas.ifsul.edu.br

(2) Centro de Desenvolvimento Tecnológico - Universidade Federal de Pelotas - CDTec-UFPel e-mail: andreiasias@pelotas.ifsul.edu.br

(3) Centro de Desenvolvimento Tecnológico - Universidade Federal de Pelotas - CDTec-UFPel e-mail: rafaelcardoso@pelotas.ufpel.edu.br

(4) Centro de Desenvolvimento Tecnológico - Universidade Federal de Pelotas - CDTec-UFPel e-mail: tatiana@inf.ufpel.edu.br

\begin{abstract}
Palavras-chave: interação Humano-computador, interface de usuário, tecnologia assistiva O artigo apresenta, através de um Mapeamento Sistemático de Literatura, o estado da arte da pesquisa sobre as diversas Interfaces de Usuário (IU) aplicadas em Tecnologia Assistiva (TA) para usuários com deficiência motora elou visual, apresentando padrões, tendências e novos espaços de pesquisa nesse campo de estudo da interação humanocomputador.
\end{abstract}

Key-words: Human-computer interaction, user interface, assistive technology The article presents, through a Systematic Literature Review, the state-of-the-art of research on User Interfaces (UI) applied in Assistive Technology (TA) for users with motor and /or visual impairment, presenting patterns, trends and new gaps of research in this field of study of human-computer interaction.

\section{Introdução}

Este artigo tem por objetivo apresentar um estudo sobre a relação da Tecnologia Assistiva (TA), com suas Interfaces de Usuário (IU), especificamente relacionadas às pessoas com deficiência visual e/ou motora.

O que se propõe é delimitar o estado da arte desse cenário, a partir de um Mapeamento Sistemático de Literatura (MSL), com análise de artigos publicados sobre essas temáticas, criando base para novas pesquisas dentro desse campo do conhecimento e na produção de TA mais efetiva e aderente às necessidades desses usuários atípicos.

O presente trabalho se estrutura na apresentação dos pilares teóricos utilizados na delimitação do estudo; na definição de protocolo e método de análise dos artigos utilizados; e na análise dos dados coletados que geraram alguns apontamentos e conclusões.

\subsection{Cenário da Tecnologia Assistiva}

Segundo dados da Organização das Nações Unidas (ONU), estima-se que $15 \%$ da população mundial (cerca de 1 bilhão de pessoas em 2011) possuíam "deficiências graves ou moderadas", enquanto 3,8\% ou cerca de 190 milhões apresentavam "deficiências graves" (WORLD HEALTH ORGANIZATION, 2011).

Conforme dados do Instituto Brasileiro de Geografia e Estatística (IBGE, 2010) há no Brasil cerca de 45,6 milhões de pessoas com deficiência, o que corresponde a $23,9 \%$ da população brasileira; deste total apresentam deficiências severas $8,3 \%$ da população, sendo que $2,33 \%$ são pessoas com 
deficiência motora severa e 3,46\% deficiência visual severa. Como é mostrado na Gráfico $1,7 \%$ da população apresenta algum tipo de deficiência motora e 18,6\% apresenta alguma deficiência visual.

Gráfico 1 - Distribuição de tipos de deficiência no Brasil

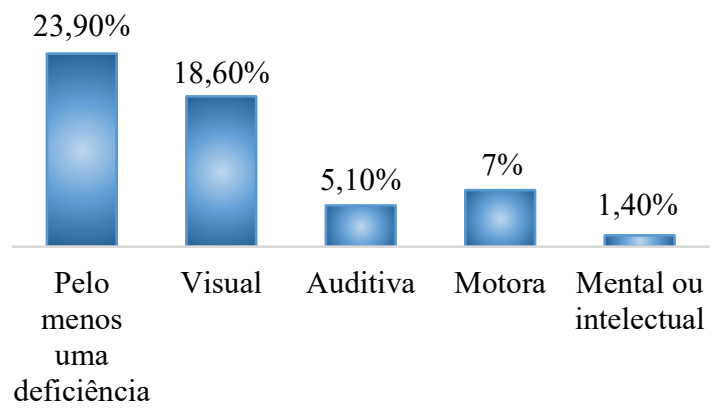

Fonte: Cartilha Senso 2010

Nesse contexto, justificam-se, portanto, os esforços na busca de soluções que contemplem esse grande grupo de usuários que ficam a margem de processos de sociabilização básicos, como ter acesso à educação, ao mundo do trabalho e de uma própria auto imagem de pessoa com capacidades iguais a qualquer outra que seja um usuário típico (SHINOHARA, WOBBROCK, 2016).

A área de TA surge como resposta a essa demanda de inclusão das pessoas com deficiência, num campo de estudo multidisciplinar que visa propiciar a real inclusão desses usuários, conferindo-os autonomia no desenvolvimento das atividades cotidianas (BERSCH, 2008).

O conceito "Tecnologia Assistiva" veio da tradução de Assistive Technology, importante termo jurídico dentro da legislação norte-americana, conhecida como Public Law 100-407 de 1988. Tal lei foi pioneira nos processos de regulação legal desse tipo de tecnologia, obrigando o governo norte-americano a disponibilizar o acesso a todo o material necessário possível para que os deficientes tivessem mais independência, sendo mais produtivos (BERSCH, 2008).

É importante compreender que uma TA não necessita estar relacionada a um dispositivo tecnológico, como computador, e sim ser um dispositivo que tenha como objetivo ampliar ou capacitar um deficiente a realizar tarefas específicas, dificultadas diante alguma limitação. Um óculos (que melhora a visão para uma pessoa com miopia), uma cadeira de rodas (que possibilita autonomia de deslocamento para um deficiente físico), um simples suporte que possibilite uma pessoa com paralisia segurar de modo mais eficiente um lápis, são exemplos de TA.

Diante desse amplo alcance de soluções possíveis dentro da área, existem diversas classificações para os tipos possíveis de TA. A ISO 9999, por exemplo, se detém mais em uma concepção técnica, classificando as diversas TA em produtos e ferramentas, utilizando classes (por exemplo, classe 18 mobiliário e adaptações para habitações; classe 06 órteses e próteses). Já a classificação HEART (Horizontal European Activities in Rehabilitation Technology) baseia-se no uso final da TA em questão, dividindo-as em componentes técnicos, humanos e socioeconômicos (por exemplo, uma TA para comunicação que possibilite acesso ao emprego) sendo um tipo de classificação menos utilizada, contudo mais aderente para abordagem aqui proposta deste estudo (OHLINE et al,. 1995).

Entender brevemente essa categorização, e os seus diversos tipos, é importante na organização, prescrição de esforços de pesquisa de TA e em como elas são tratadas nos artigos científicos.

O foco desse artigo reside na intersecção da criação da TA com o uso das Tecnologias da Comunicação e Informação (TICs), permitindo acessibilidade ao uso de: computadores, tablets, smarthphones, consoles de jogos eletrônicos, entre outros diversos dispositivos que estão em nossas atividades de lazer, trabalho e educação.

Nesse caso, as TICs podem ser utilizadas ou como TA, ou através de uma TA, e propiciam real inclusão a novos contextos (BERSCH, 2008, p. 2), como por exemplo, acesso à educação em ambientes virtuais de aprendizagem.

\subsection{Interfaces de Usuário (IU)}

Pensar interfaces dentro do campo de estudo da Interação Humano-Computador (IHC) é refletir sobre a ponte de comunicação entre máquinas e os usuários (ROYO, 2008). São elas as tradutoras de 
linguagens computacionais (JOHNSON, 2001) que possibilitam a interação e manipulação de dados em diversos níveis através das novas Tecnologias de Informação e Comunicação, as quais são importantes instrumentos de nossa cultura e, sua utilização por todos, um meio concreto de inclusão no mundo (LÉVY, 2004, p. 27).

Historicamente o desenvolvimento das interfaces, que possibilitam a Interação Humano-Computador, ocorreram dentro de paradigmas criados na própria evolução tecnológica dos dispositivos. Se no início da era computacional era mais simples falarmos a linguagem das máquinas através de uma Interface de Usuário com linha de comando inserida através do teclado (Interface baseada em comandos), hoje dispõe-se de uma infinidade de possibilidades de interação mais próximas a linguagem do usuário, como por exemplo, através de dispositivos que monitoram um simples balançar de nossa cabeça (gesto / Interface natural), ou de uma fala (voz / Interface por comandos de voz) como uma ação de input para um determinado comando de output de uma dada TIC (PREECE et al., 2013).

No entanto, grande parte do paradigma atual adotado como Interface de Usuário, para relacionamento com os sistemas computacionais, baseia-se na Interface Gráfica de Usuário (IGU). A IGU surgiu como facilitadora no processo de difusão do computador como ferramenta de trabalho e lazer, através do uso do computador pessoal, pois utiliza metáforas visuais gráficas como base de interação, como por exemplo, para exclusão de um arquivo basta movimentá-lo para o ícone da lixeira.

Através dessas metáforas de uso, com janelas, ícones, menus e dispositivos de apontar (paradigma WIMP, Windows, Icons, Menus e Pointers), as Interfaces de Usuário que se baseiam em Interfaces Gráficas utilizam movimentação de cursor para seleção e clique (PREECE et al., 2013). Para tanto popularizou-se o uso de alguns dos dispositivos de input mais utilizados até hoje, que são o mouse e o teclado, como básicos na interação com o computador, além das telas em diversos formatos como dispositivos output que geram as respostas visuais a nossas interações no sistema.

Mesmo nos dispositivos que utilizam a tecnologia de toque na tela (touchscreen) fica caracterizado, pelo uso de um apontador (ponta dos dedos) como ferramenta de interação, a condição de uma Interface Gráfica do Usuário. Usuários com restrições de movimento nos membros superiores, com deficiência visual, enfrentam grandes barreiras de utilização desse tipo de interface (NICOLAU et al., 2014).

Percebemos então, que as características humanas são fator determinante no modo como se estrutura a relação de uso das TICs através desses diversos tipos de interfaces. Essa relação nada mais é do que o processo de interação, ou seja, a troca de estímulos e respostas que colocamos (input) e recebemos (output) dos sistemas computacionais e que hoje ainda dependem dos sentidos básicos do nosso corpo: como visão, audição, tato e da capacidade de movimentar o corpo (BARBOSA, SILVA, 2010).

Nesse contexto, usuários com alguma deficiência ficam excluídos da utilização da maioria dos tipos das IU, já que precisam de dispositivos auxiliares que possibilitem selecionar, clicar, arrastar e interagir com essas interfaces (RODRIGUES; ALVES, 2013).

Nesse ponto a importância de se dispor de uma TA adequada para cada tipo de deficiência é o que vai definir o uso dessa ou daquela Interface de Usuário, dado que mesmo com todo a acessibilidade embarcada na maioria dos sistemas operacionais padrões de mercado, ainda existem diversas barreiras de manipulação dessas interfaces (PLOS et al., 2012).

\section{Metodologia}

Utilizou-se o método do Mapeamento Sistemático de Literatura ou MSL (PETERSEN et al., 2008) como base para o desenvolvimento deste estudo. Essa metodologia consiste na ideia de uma análise exploratória através de engenhos de busca, em bases consolidadas de artigos científicos, seguindo protocolos específicos, os quais possibilitam a criação de uma massa crítica de entendimento sobre trabalhos similares ou de norteadores gerais para aplicação na pesquisa de uma determinada área.

Uma característica importante da MSL é que todos os procedimentos executados devem ser documentados, de maneira que o estudo realizado 
possa ser reproduzível por outros pesquisadores, de forma que estes consigam alcançar os mesmos resultados encontrados no período da pesquisa (RAVANELLO et al., 2016). Com a aplicação deste processo de pesquisa, espera-se minimizar ou justificar a ausência de trabalhos que poderiam ser considerados importantes dentro levantamento bibliográfico feito.

Dentro do objetivo proposto neste trabalho delimitou-se o uso do MSL as suas fases iniciais de a) definição de questões gerais de pesquisa e b) de análise geral de artigos relevantes.

O protocolo mínimo definido para esse estudo foi a utilização de uma busca de artigos científicos através do engenho específico Google Scholar (http://scholar.google.com.br/). Esta escolha é justificada pois este engenho indexa todas as demais bases de dados recorrentes de pesquisa em artigos, não sendo restritiva a uma área específica, permitindo disponibilidade facilitada de acesso.

Foram consideradas publicações realizadas entre 2011 a 2016, somente no idioma inglês e que utilizavam dentro de suas palavras chaves, título ou do seu resumo os termos:

HCI; interface; user interface; assistive technology; accessible; adaptive; assistive; accessibility; visual impaired; motor impaired; disability; device interaction;

Como critérios para exclusão de um artigo para análise, foi definido que ele não poderia ser relacionado a área da educação, ou específico com abordagem de área médica (utilização em conjunto das palavras-chave education e/ou medical).

Outro fator de seleção e/ou exclusão foi a opção de somente analisar artigos relacionados a deficiência motora e visual, já que ambos encontram maior representatividade dentro do universo de usuários atípicos (IBGE,2010).

No protocolo foram definidas duas perguntas de pesquisas gerais:

Q1: Que modos de interação são mais comumente utilizados por pessoas com deficiência visual e motora para utilizar o computador?

Q2: Que tipos de dispositivos e softwares são utilizados para interação humano-computador por pessoas com essas deficiências?

Estas perguntas nortearam a análise dos artigos resultantes da aplicação do protocolo, através de leitura dos seus resumos. Alguns artigos nos quais o resumo não responde essas perguntas optou-se pela leitura completa ou parcial dos mesmos.

\section{Resultados e discussões}

A aplicação do protocolo (novembro de 2016) gerou um conjunto extenso de artigos (327 no total), que foram analisados dentro das questões gerais elencadas e, desse modo, se propôs uma discussão qualitativa sobre o material coletado, desenvolvendo uma análise exploratória sobre os temas.

Um dos primeiros aspectos relevantes é que muitos dos artigos retornados tratam da relação da TA não com uma deficiência específica, mas sim em seu uso por pessoas idosas com algumas limitações (CALLARI et al., 2012). Isso fez com que esses artigos fossem considerados aptos para análise, desde que tivessem TA para acessibilidade de TICs entre seus objetivos.

Outro ponto que despertou atenção foi a distribuição dos artigos publicados resultantes da busca durante o intervalo de anos pesquisados (Tabela 1), evidenciando o crescente número de pesquisas relacionadas ao assunto nos últimos anos.

Já com relação aos tipos de deficiência que utilizam uma determinada TA, constatou-se um amplo espectro de limitações, com tipos diversos de deficiência que as pessoas apresentam.

É mister ter o entendimento desse contexto variável dos usuários atípicos, pois dentro de cada uma dessas limitações existem nuances, desde restrições leves (como uma visão parcial, miopia, dificuldade de controle do movimento de uma das mãos) até outras mais severas (cegueira completa, doenças degenerativas que afetam a capacidade motora, como Esclerose Lateral Amiotrófica) que dificultam até a capacidade de comunicação da pessoa 


\section{(BLIGNAUT, 2016).}

Tabela 1 - Artigos publicados, segundo protocolo, sobre TA com foco em acessibilidade para deficientes visuais e motores nos últimos 5 anos.

\begin{tabular}{lc}
\hline Ano & Quantidade de artigos publicados \\
\hline 2011 & 11 \\
\hline 2012 & 37 \\
\hline 2013 & 63 \\
\hline 2014 & 52 \\
\hline 2015 & 61 \\
\hline 2016 & 66 \\
\hline Total & $\mathbf{2 9 0}$ \\
\hline
\end{tabular}

Nesse sentido, dentro do universo de possíveis soluções de TA mapeadas, segundo o enfoque de classificação de dispositivos de auxílio no uso do computador, os artigos analisados podem ser divididos, em linhas gerais, em dois grandes grupos:

Artigos descritivos - apresentam algum software/dispositivo que adapta as Interfaces de Usuário das Tecnologias de Informação e Comunicação tradicionais, tentando compensar a

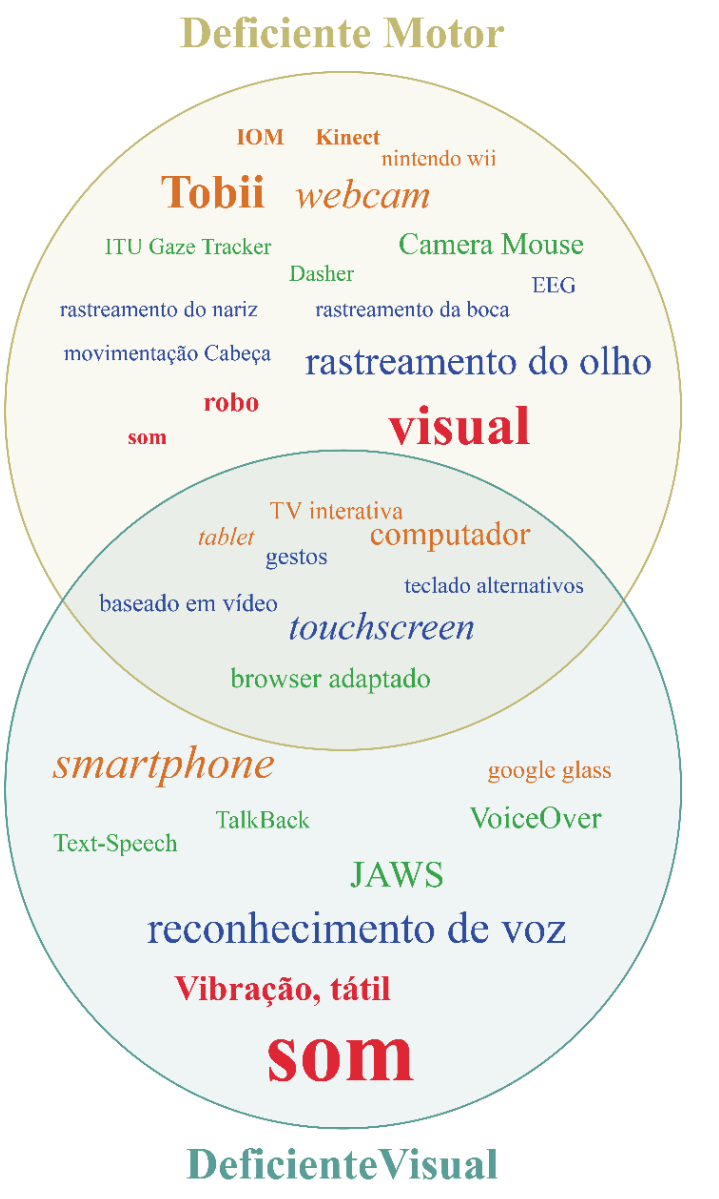

Legenda: deficiência pelo uso de uma Tecnologia Assistiva;

Artigos analíticos - baseados em análise/pesquisa comparativa entre o uso de várias TAs com a mesma finalidade, avaliando a eficiência no uso em relação a IU.

Gráfico 2 - Ocorrências de modos de interação/dispositivos/softwares, input e output de dados vs. tipo de deficiência nos artigos retornados

Caracterizou-se que cada tipo de deficiência, dada sua especificidade, demandou um tipo de abordagem diferente na maneira como a interação com o computador se estabelece - utilizando dispositivos de TA para input e output de dados (BARBOSA, SILVA, 2010).

Com base nessas considerações, e na análise qualitativa dos artigos, criou-se um diagrama representativo (Gráfico 2), no qual temos os círculos representando as duas grandes áreas de deficiência (visual e motora) e, dentro dessas, as ocorrências dos modos de interação, dispositivos e softwares citados classificados segundo a legenda.

A partir desse diagrama é possível traçar considerações gerais sobre o espectro das Interfaces de Usuário atreladas à Tecnologia Assistiva e as deficiências:

De uma maneira geral percebe-se um espectro mais amplo de interações propostas no uso das TICs/TAs através das IU para os deficientes com deficiência motora, pois existem vários níveis de comprometimento físico envolvidos nesse caso, o que gera interfaces personalizadas; enquanto que a deficiência visual, apesar de ter também vários níveis de acuidade (desde a cegueira completa até visão parcial), possui uma uniformidade maior nos tipos de tratamento das interfaces que ficam baseadas principalmente em voz e háptica.

\subsection{Interfaces de Usuário para deficiência motora}

Os principais modos de interação referenciados junto às IU através das TA (Q1) são as baseadas em rastreamento de movimento (principalmente olhos e cabeça, mas também língua, boca e nariz) (HUO et al., 2013) (RODRIGUES et al., 2016). Nesse sentido os dispositivos potencializam o controle do cursor do mouse através de tecnologias vestíveis (como 
pulseiras e óculos adaptados) e de rastreamento de imagem (através de gestos - Interface Natural), sendo os mais citados (Q2) o software Camera Mouse e o dispositivo de captação de imagens Tobii, ambos utilizados diretamente no computador.

Em menor grau foram citados teclados adaptados (one push button, por exemplo) (KURSCHL et al., 2013) ou apêndices que possibilitam o uso de smartphone com interação por toque. A maioria do output de dados nesse tipo de deficiência é dada através de interfaces gráficas (IGU) dos sistemas nativos dos computadores ou adaptações desses (como browser ou aplicativos de uso de TV interativa específicos).

A grande utilização do uso de recursos do próprio computador, como a webcam, é claramente uma resposta às pesquisas que utilizam o leitor de imagens Tobii, que é um produto de mercado, com custo elevado, o que inviabiliza seu uso pela maioria das pessoas com deficiência. Aponta-se, com isso, numa característica da maioria das pesquisas irem em busca de soluções de baixo custo e que utilizem, com poucas adaptações, os próprios recursos disponíveis pela maioria das pessoas (BISWAS et al., 2013).

Nesse sentido as soluções de rastreamento de movimento dividem-se em soluções não invasivas (rastreamento de movimento de cabeça, olhos) e outras que são invasivas (uso da língua para uso de joystick, por exemplo) sem uma boa experiência de uso (questões de higiene, praticidade de uso) como dispositivo de interação com as interfaces.

\subsection{Interfaces de Usuário para deficientes visuais}

A grande base de interações estabelecidas com as TICs nas IUs é relacionada a (Q1) input e output de comandos de voz. Interfaces hápticas (táteis) também foram bastante citadas e geram reconhecimento de componentes que normalmente seriam visuais (GIUDICE, 2012), porém com uma tradução para vibração ou som (EL SADDIK et al., 2011).

Em menor quantidade foram citados teclados adaptados com braille, normalmente conectados a (Q2) computadores, enquanto que os smarthphones e tablets também foram muito utilizados como suporte de TA para localização especial dentro de um determinado ambiente. Os softwares com maior ocorrência nos artigos verificados foram o JAWS, VoiceOver e TalkBack, sendo os dois últimos ligados a plataformas móveis mais usadas, iOS e Android (CSAPÓ et al., 2015).

As pesquisas focam principalmente no uso de dispositivos móveis para condição de guia no auxílio à mobilidade urbana (LAHAV, 2014) (RODRIGUEZ-SANCHEZ et al.,2014).

\subsection{Tendências de pesquisa em Tecnologia Assistiva e Interface de Usuário}

Mesmo dispondo de soluções tecnologicamente viáveis, e até disponíveis no mercado, para uso de IU através de TA, novas tendências de pesquisa hoje direcionam esforços em soluções ainda não maduras, do ponto de vista comercial, mas promissoras enquanto agentes de inclusão no uso das TICs.

Como tendência de novas formas de interação aparecem pesquisas com Interface CérebroComputador (Brain Computer Interface - BIC) com captura de ondas cerebrais através de EEG (eletroencefalografia).

Através da facilidade de acesso a sensores diversos e na melhoria de captação de sinais desses, os estudos não se limitam exclusivamente no uso de sinais cerebrais, mas também no uso de sinais musculares diversos com uso de EMG (EletroMioGrafia) e EOG (EletroOculoGrafia) (MÜLLER-PUTZ et al., 2015) (PINHEIRO et al., 2011).

Essa abordagem de interação mediada por sinais fisiológicos cria toda uma nova plataforma de aplicações e potencializa a TA, dado que possibilita que pessoas com restrições severas de movimento possam, através de partes do seu corpo, manipular dados através de interfaces mais customizadas com os sistemas computacionais (CARUSO et al., 2013). Uma das principais desvantagens destes sistemas é a dificuldade de extrair o sinal de interação, uma vez que a taxa de ruído de sinal (TRS) é muito baixa. Outras desvantagens importantes destes sistemas são baixa portabilidade e exigência de hardware altamente especializado (KALUNGA et al., 2014). 


\section{Considerações finais}

Através desse estudo, com base nos artigos levantados através do MSL, percebe-se que muitas pesquisas relacionadas ao tema, contudo ainda existem lacunas de investigação na área de Interação Humano-Computador relacionada a pessoas com deficiência.

Com relação ao grupo de artigos descritivos percebeu-se que comercialmente as soluções de Interfaces de Usuário dos dispositivos computacionais, em geral, são criadas para os usuários típicos, o que demanda uma série de adaptações ou de TA específica que permita o mínimo de acessibilidade aos deficientes.

A dificuldade inerente a quantidade diversa de níveis de deficiência é determinar um protocolo geral padrão de desenvolvimento de projetos que abarque a maioria das restrições, o que gera soluções de TA sob demanda para cada usuário atípico. Isso torna qualquer Tecnologia Assistiva um produto dispendioso, e que fica fora do alcance do grande grupo de pessoas que necessitam daquela ferramenta.

Com relação ao grupo de artigos analíticos o processo de avaliação e comparação entre dispositivos/softwares utilizados como TA apresentaram grandes disparidades, apontando que não existe uma padronização tanto de desenvolvimento de uma Tecnologia Assistiva, bem de avaliação de uso desta pelo seu perfil de usuário.

A partir dessas considerações é possível elencar pesquisas futuras dentro desse escopo de Interfaces de Usuário e Tecnologia Assistiva:

- Criação de tecnologias que considerem suas Interfaces de Usuário baseadas no Design Universal, que seja inclusivo como base e não como apêndice;

- Desenvolvimento de dispositivos de TA multimodais que suportem mais de um input sensorial de dado, como áudio, movimento de cabeça, sendo deste modo possível que usuários com diversos tipos de deficiência sintam-se contemplados;

- Definição de protocolos de avaliação mais abrangentes que avaliem não somente a usabilidade técnica desses dispositivos, mas também sua experiência de uso;

\section{Referências bibliográficas}

AL-RAHAYFEH, A., e FAEZIPOUR, M. Eye Tracking and Head Movement Detection: A State-of-Art Survey. IEEE Journal of Translational Engineering in Health and Medicine, 2013.

\section{BARBOSA, S.D.J., SILVA, B.S. Interação}

Humano-Computador. Rio de Janeiro: Elsevier, 2010.

BERSCH, R. Introdução a Tecnologia Assistiva. CEDI: Centro Especializado em Desenvolvimento Infantil: Porto Alegre, 2008. Disponível em $<$ http://www.assistiva.com.br/Introducao_Tecnologi a_Assistiva.pdf $>$. Acesso em: 16 ago. 2015

BISWAS, P., ROBINSON, P. e LANGDON, P. Designing inclusive interfaces through user modeling and simulation. International Journal of Human-Computer Interaction, v. 28, n. 1, p. 1-33, Londres. 2012.

\section{BISWAS, P., e LANGDON, P. Multimodal} intelligent eye-gaze tracking system. International Journal of Human-Computer Interaction, 31(4), 277 294. Londres, 2015.

BISWAS, P., JOSHI, R., CHATTOPADHYAY, S., ACHARYA, U. R., e LIM, T. C. Interaction techniques for users with severe motorimpairment. A Multimodal End-2-End Approach to Accessible Computing (pp. 119-134). Springer Londres. 2013.

BLIGNAUT, P. Development of a gaze-controlled support system for a person in an advanced stage of multiple sclerosis: a case study. Universal Access in the Information Society, p. 1-14. 2016

BRASIL, Subsecretaria Nacional de Promoção dos Direitos da Pessoa com Deficiência. Comitê de Ajudas Técnicas. Tecnologia Assistiva. - Brasília : CORDE, 2009.

CARUSO, M., CINCOTTI, F., LEOTTA, F., MECELLA, M., RICCIO, A., SCHETTINI, F., e 
CATARCI, T. My-world-in-my-tablet: an architecture for people with physical impairment. International Conference on Human-Computer Interaction (pp. 637-647). Springer Berlin Heidelberg.2013.

CALLARI, T. C., CIAIRANO, S., e RE, A. Elderly-technology interaction: accessibility and acceptability of technological devices promoting motor and cognitive training. Work, 41(Supplement 1), 362-369. 2012.

CSAPÓ, Á., WERSÉNYI, G., NAGY, H., e STOCKMAN, T. A survey of assistive technologies and applications for blind users on mobile platforms: a review and foundation for research. Journal on Multimodal User Interfaces, 9(4), 275-286. Londres. 2015

EL SADDIK, A., OROZCO, M., EID, M., e CHA, J. Haptics: Haptics Applications. In Haptics Technologies (pp. 21-43). Springer Berlin Heidelberg. 2011.

GIUDICE, N. A., PALANI, H. P., BRENNER, E., e KRAMER, K. M. Learning non-visual graphical information using a touch-based vibro-audio interface. Proceedings of the 14th international ACM SIGACCESS conference on Computers and accessibility (pp. 103-110). ACM. 2012.

HUO, X., PARK, H., KIM, J., e GHOVANLOO, M. A dual-mode human computer interface combining speech and tongue motion for people with severe disabilities. IEEE Transactions on Neural Systems and Rehabilitation Engineering, 21(6), 979-991. 2013.

IBGE, Instituto Brasileiro de Geografia e Estatística. Censo Demográfico. 2010. Disponível em < http://www.ibge.gov.br/home/estatistica/populacao/c enso2010> Acesso em 4 ago. 2015

\section{INTERNATIONAL ORGANIZATION FOR} STANDARDIZATION: ISO 9999:2002 Assistive products for persons with disability -

Classification and terminology. Genebra, 2001.

JOHNSON, S. Cultura da interface: como o computador transforma nossa maneira de criar e comunicar. Rio de Janeiro: Jorge Zahar Ed., 2001.
KURSCHL, W., AUGSTEIN, M., STITZ, H., HEUMADER, P., e POINTNER, C. A user modelling wizard for people with motor impairments. Proceedings of International Conference on Advances in Mobile Computing \& Multimedia (p. 541). ACM. 2013.

KALUNGA, E. K., CHEVALLIER, S., RABREAU, O., e MONACELLI, E. Hybrid interface:

Integrating BCI in multimodal human-machine interfaces. In Advanced Intelligent Mechatronics (AIM), 2014 IEEE/ASME International Conference on (pp. 530-535). IEEE. 2014.

LAHAV, O. (2014). Virtual reality as orientation and mobility aid for blind people. Journal of Assistive Technologies, 8(2), 95-107.

LÉVY, P. As tecnologias da Inteligência - O futuro do pensamento na era da informática. 13.ed. São Paulo: Editora 34, 2004.

LUPU, R. G., e UNGUREANU, F. A survey of eye tracking methods and applications. Mathematics Subject Classification:68U35,68N19,94A12, LXIII(3), 72-86. 2013.

MÜLLER-PUTZ, G., LEEB, R., TANGERMANN, M., HÖHNE, J., KÜBLER, A., CINCOTTI, F., E MILLÁN, J. D. R. Towards noninvasive hybrid brain-computer interfaces: framework, practice, clinical application, and beyond. Proceedings of the IEEE, 103(6), 926-943. 2015.

NICOLAU, H. Mobile touchscreen user interfaces: bridging the gap between motorimpaired and able-bodied users. Universal access in the information society, v. 13, n. 3, p. 303-313, 2014.

OHLINE, P., FAGERBERG, G., e LAGERWALL, $T$., Technology for Assisting Disabled and Older People in Europe: The Heart Study. TIDE. Londres, 1995.

PETERSEN, K., FELDT, R., MUTJABA, S. e MATTSSON, M., Systematic mapping studies in software engineering, Proceedings of the 12th International Conference on Evaluation and Assessment in Software Engineering (EASE), 2008. 
PINHEIRO, C. G., NAVES, E. L., PINO, P., LOSSON, E., ANDRADE, A. O., e BOURHIS, G. Alternative communication systems for people with severe motor disabilities: a survey. Biomedical engineering online, 10(1), 31. 2011.

PLOS, O. A Universalist strategy for the design of Assistive Technology. International Journal of Industrial Ergonomics, v. 42, n. 6, p. 533-541, 2012.

PREECE, J.; ROGERS, I.; SHARP, H. Design de Interação: Além da Interação HumanoComputador. Porto Alegre: Bookman, 2013.

RAVANELLO, I. M., WOLFF, F. e RIBEIRO, V. G.,Uma revisão sistemática da produção bibliográfica sobre Experiência de Usuário no Campo do Design. Ergodesign \& HCI, Rio de Janeiro, v. 4, ano 4, 2016.

RODRIGUES, P. R.; ALVES, L. R. G.. Tecnologia Assistiva - Uma Revisão Do Tema. HOLOS, Natal: v. 29, n. 6, p. $170-180,2013$.

RODRIGUES, A. S., DA COSTA, V., MACHADO, M. B., ROCHA, A. L., DE OLIVEIRA, J. M., MACHADO, M. B., E TAVARES, T. A.

Evaluation of the use of eye and head movements for mouse-like functions by using IOM device. In Lecture Notes in Computer Science (including subseries Lecture Notes in Artificial Intelligence and Lecture Notes in Bioinformatics) (Vol. 9738, pp. 81-91). Springer Verlag. 2016.

RODRIGUEZ-SANCHEZ, M. C., MORENOALVAREZ, M. A., MARTIN, E., BORROMEO, S., e HERNANDEZ-TAMAMES, J. A. Accessible smartphones for blind users: A case study for a wayfinding system. Expert Systems with Applications, 41(16), 7210-7222. 2014.

ROYO, J. Fundamentos do Design Digital. São Paulo: Edições Rosari, 2008.

SHINOHARA, K.; WOBBROCK, J. O. Selfconscious or self-confident? a diary study conceptualizing the social accessibility of assistive technology. Transactions on Accessible Computing (TACCESS)., v. 8, n. 2, p. 5:1-5:31. New York, NY, EUA, 2016.
World Health Organization (WHO): The World Bank: World Report of Disability. WHO Press, World Health Organization, Genebra, 2011.

World Health Organization (WHO): International Classification of Functioning, Disability and Health: ICF. WHO Press, World Health Organization, Genebra, 2001. 